\title{
Research on Noise Removal in Fiber Grating Sensing Signal
}

\author{
Xiaobo Zhou ${ }^{1, a^{*}}$ \\ ${ }^{1}$ Xijing university, Xi'an, china \\ a369146317@qq.com
}

Keywords: Fiber grating; Sensing signal; Noise interference; Contourlet transform; Filter

\begin{abstract}
Signal processing is one of the key technology of fiber grating sensor, noise interference on signal transmission, influence of fiber grating sensor practical application effect, in order to solve the noise problem of fiber grating sensing signal, design a denoising method of fiber grating sensing signal based on improved contourlet transform. The acquisition of fiber grating sensing signal firstly, and remove the unwanted signal, select the useful signal, then using contourlet transform, by filtering the noise filter, finally through the concrete experiment to test the effect of denoising. The results show that the improved contourlet transform can completely remove the fiber Bragg grating sensing signals, and improve the quality of FBG sensor communication, and verify the effectiveness of the proposed method.
\end{abstract}

\section{Introduction}

With the continuous development of optical communication technology, fiber grating sensing technology obtained the unprecedented development of fiber Bragg grating sensor with strong ability of resisting electromagnetic interference resistance to corrosion, high sensitivity, small body Low cost advantages, has the incomparable advantages of traditional sensors, in the national defense military aerospace and other fields has been widely used in [1-3] in the practical application of fiber Bragg grating sensor and sensing signal is very susceptible to noise interference, the measurement precision of the fiber Bragg grating sensor is affected, so to remove the noise of the fiber Bragg grating sensor research has the vital significance.

At home and abroad in recent years, some studies mechanism of fiber Bragg grating sensor signal denoising studies $[7,8]$, but the study also not enough in-depth the earliest using Fourier transform to deal with the noise of the fiber Bragg grating sensor signal, in order to improve the quality of the fiber Bragg grating sensor signals, but as a result of the Fourier transform of signal decomposition scale is coarser, cannot effectively eliminate the noise of the fiber Bragg grating sensor signal [9-11] In order to solve the limitations of Fourier transform, some scholars wavelet transform denoising method was adopted to realize FBG sensing signal quality of ascension, the decomposition scale is more detailed, more thoroughly remove noise, but the algorithm of wavelet threshold denoising process, the threshold selection is critical, whether hard or soft threshold threshold was adopted to realize, the current disagreed on threshold selection criteria, and the implementation process is very complicated, not easy to operation.

Outline of the wavelet transform is a method of multi-scale decomposition can effective denoising signal in noise, in order to solve the problem of the current fiber Bragg grating sensor signal noise, design a fiber Bragg grating sensor based on contour wavelet transform signal denoising method, the experimental results, improve the outline of the wavelet transform can eliminate noise in the fiber Bragg grating sensor signals, won the high quality of the grating sensor signal. 


\section{Working Principle of Fiber Grating Sensing}

Fiber Bragg grating sensor is a kind of wavelength modulated sensor. As the wavelength is a constant, it is not affected by the optical fiber loss and energy of the light source, so its performance is better than other types of fiber sensor. Its working principle is shown in figure 1.

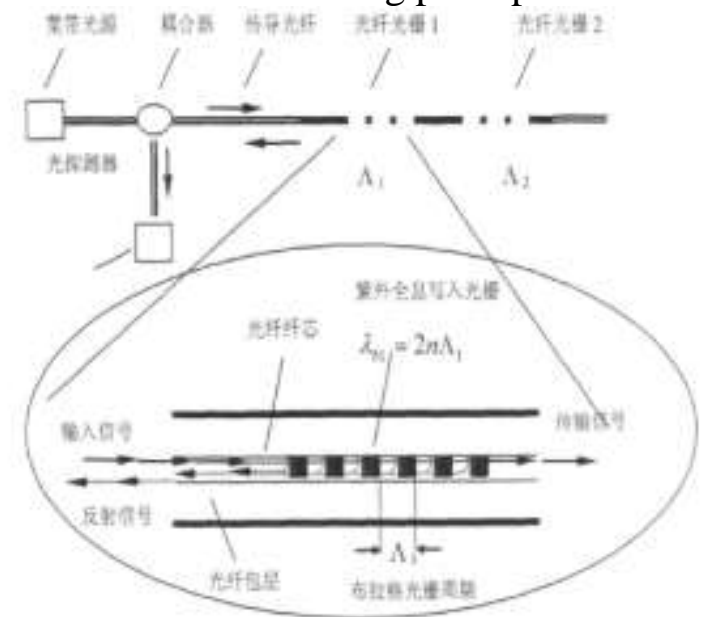

Figure 1. Working Principle of Fiber Grating Sensing

appens, reflection wavelength will have corresponding change, so that we can according to the detection wavelength to be the measured value of the fiber grating, such as temperature and pressure center wavelength drift $(\Delta \lambda)$ and the relationship between the longitudinal strain $(\Delta \varepsilon)$ can be described as

$$
\frac{\Delta \lambda_{B}}{\lambda_{B}}=\left(1-P_{e}\right) \Delta \varepsilon
$$

$P_{e}=\frac{1}{n} \times d n / d \varepsilon$ denotes the elastic modulus.

The relationship between the center wavelength drift $(\Delta \lambda)$ and temperature change $(\Delta T)$ of the FBG can be described as

$$
\frac{\Delta \lambda_{B}}{\lambda_{B}}=\left(\alpha_{t}-\xi\right) \Delta T
$$

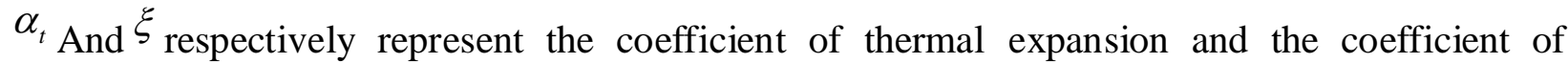
thermal light.

The relationship between the center wavelength drift ( $\Delta \lambda)$ and pressure change $(\Delta P)$ of the fiber Bragg grating is

$$
\frac{\Delta \lambda}{\lambda}=\frac{\Delta(n \Lambda)}{n \Lambda}=\left(\frac{1}{\lambda} \times \frac{\partial \Lambda}{\partial P}+\frac{1}{n} \times \frac{\partial n}{\partial P}\right) \Delta P
$$

Let $\mathrm{E}$ be the elastic modulus of the fiber Bragg grating, and the formula for calculating the refractive index change of the fiber Bragg grating is

$$
\begin{aligned}
\frac{\Delta L}{L} & =\frac{(1-2 v) P}{E} \\
\frac{\Delta n}{n} & =\frac{n^{2} P}{2 E}(1-2 v)\left(2 \rho_{12}+\rho_{11}\right)
\end{aligned}
$$

In the process of fiber Bragg grating sensing, the refractive index is related to many factors, especially the interference of noise is the biggest, which brings adverse effects to the measurement results such as temperature and pressure. For this reason, contour transformation is introduced in 
this paper to carry out denoising processing on the fiber Bragg grating sensing signal, so as to improve the measurement accuracy such as temperature and pressure.

\section{The Method of Raster Sensing Signal Denoising by Contourlet Transform}

\section{Contourlet Transform}

Aiming at the limitation of the wavelet transform, some scholars raised the outline of the wavelet transform, this method can from two aspects of measure frequency, by the signal processing has a certain direction, make the effective signal to concentrate for a signal containing noise, outline of the wavelet transform of the original signal by base structure approaching, outline of the wavelet transform at the same time also has a certain anisotropic characteristics through the Laplacian pyramid to multi-scale decomposition of noise signal, find some of the specific signal and noise, through the direction of the filter to filter noise operation, after denoising signal Firstly, the fiber Bragg grating sensor signal is sampled and the low frequency information of the fiber Bragg grating sensor signal is obtained. Then, the fiber Bragg grating sensor signal is sampled, and the high frequency information of the fiber Bragg grating sensor signal is obtained. In figure 2 , X represents the fiber Bragg grating sensor signal, and $\mathrm{H} \mathrm{G}$ is a low-pass filter.

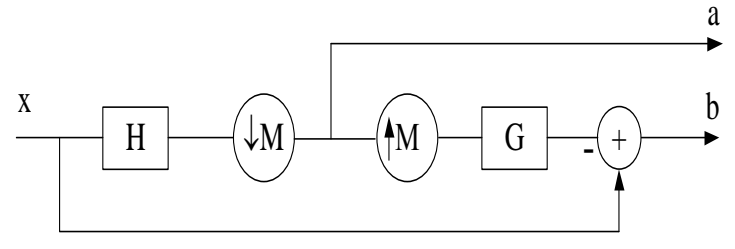

(a) Decomposition of fiber Bragg grating signals

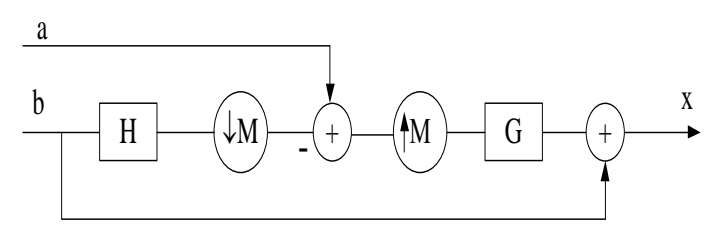

(b) Reconstruction of fiber Bragg grating signals

Figure 2. Diagram of decomposition and reconstruction of fiber Bragg grating signals

The process of three-level directional subband division of direction filter is shown in figure 3 . The sub-bands of the upper level are sampled by resampling operator and the sampling results are output.

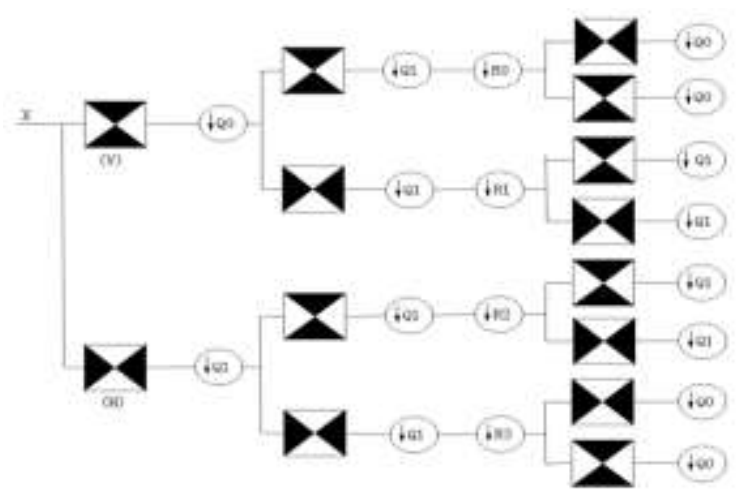

Figure3. Subband division of direction filter

The De-noising Principle of Fiber Bragg grating (FBG) Sensor in Contour Wave Transform As the useful FBG sensor signal and noise show different characteristics in contour wave transformation, the FBG sensor signal is transformed in different levels, and the noise in the FBG sensor signal is removed according to the appropriate threshold value.

Set the original fiber Bragg grating sensor signal $\{g(x) ; x=1,2, \cdots, N\}$, Signal with noise fiber grating sensor $\{f(x)=g(x)+N(x) ; x=1,2, \cdots, N\}$, Where, the mean value of noise is 0 , the variance is $\sigma^{2}$, and the formula for calculating the standard deviation of each layer is

$$
\hat{\sigma}(j)=\frac{\underset{j=1,2, \cdots, J}{\operatorname{Median}}\left(\left|w_{f}(j)\right|\right)}{0.5}
$$

$w_{f}(j)$ means the decomposition coefficient of the $f(x)$ JTH layer. 


\section{Selection of Threshold Value of Signal Denoising by Fiber Bragg Grating Sensor}

There are many threshold selection methods for contour wave transformation. Due to the universal and easy implementation of the Universe threshold method, this paper selects the Universe threshold method to determine the threshold of the optical fiber grating sensor signal denoising, and the high-frequency sub-band threshold of the Universe threshold method is determined as follows

$$
T H(j)=\sigma(j) \sqrt{2 \lg (M(j) * N(j))}
$$

Where, $M(j) * N(j)$ represents the number of contour wave decomposition coefficients of $\mathrm{j}$ layer.

$M(j) * N(j)$,the larger the size, the denoising threshold will set the high frequency coefficient of the decomposition result of the fiber Bragg grating sensor to 0 . In this way, a large number of useful fiber Bragg grating sensor signals will be removed. Therefore, equation (7) will be corrected as follows

$$
T H(j)=T H(j) / \sqrt{M(j) * N(j)}
$$

\section{The Process of Signal Denoising by Fiber Bragg Grating Sensor}

Step1: contour wave transform is performed on signal $\mathrm{f}(\mathrm{x})$ of fiber Bragg grating sensor with noise. The coefficients of contour transform domain are as follows:

$$
w=C T(f(x))=C T_{-} f+C T_{-} n
$$

Step2: according to the denoising rules, the contour transformation coefficient $(\hat{w})$ of the original image $\mathrm{g}(\mathrm{x})$ is obtained by applying the denoising rules to the original optical fiber grating sensor signal $\mathrm{w}$. The denoising rules are as follows:

$$
\hat{w}=\left\{\begin{array}{lll}
w, & \text { if } & |w| \geq T H(j) \\
0, & \text { if } & |w|<T H(j)
\end{array}\right.
$$

Step3: perform contour wave reconstruction and transformation on the estimated value $\hat{w}$ of contour wave transform coefficient, and obtain the signal of fiber Bragg grating sensing $g^{\prime}(x)$ after de-noising.

$$
g^{\prime}(x)=C T^{-1} \hat{w}
$$

\section{Test and Analysis of The Denoising Effect of Fiber Bragg Grating Sensor}

\section{The Signal of Fiber Grating Sensor With Noise}

In order to analyze the denoising effect of the fiber Bragg grating sensing signal of contour wave transform, Matlab 2014 was used as the test platform to conduct the denoising experiment. The signal of fiber grating sensor with noise is shown in figure 4.

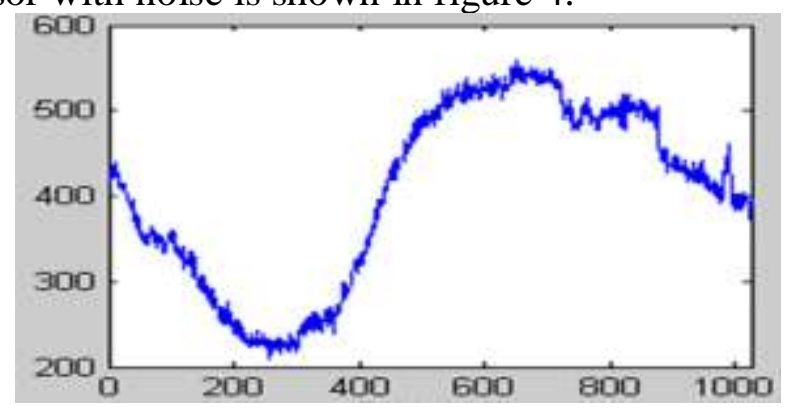

\section{Results and Analysis}

Figure4.The signal of fiber grating sensor with noise

The denoising effect of this paper.Contour wave transform is used to de-noising the signal-sensing signal of the fiber Bragg grating containing noise in figure 4. The signal-sensing signal of the fiber Bragg grating after de-noising is shown in figure 5. As can be seen from figure 5, contour wave transform can remove the noise in the fiber Bragg grating sensing signal very well and improve the quality of the fiber Bragg grating sensing signal. It is an effective method to remove noise from the fiber Bragg grating sensing signal. 


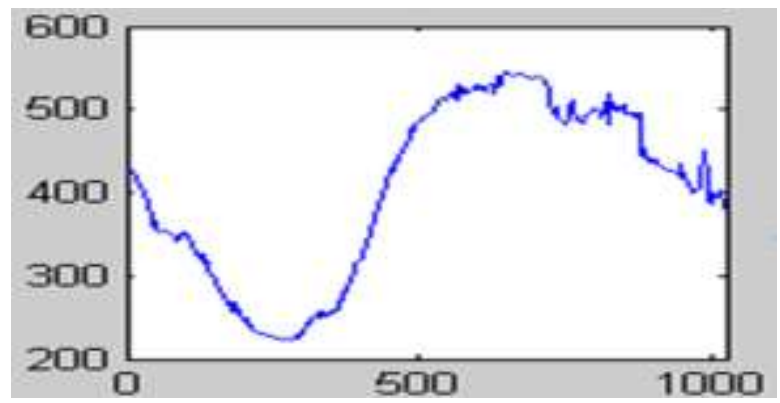

Figure5.The denoising results of fiber Bragg grating sensor in contour wave transform

Performance Comparison with Current Classical Methods.In order to test the advantage of contour wave transform in denoising, wavelet transform [16] and literature ${ }^{[17]}$ were used for comparison experiments, and peak signal to noise ratio (PSNR) and root mean square error $(\mathrm{MSE})^{[18]}$ were used to evaluate the result of signal de-noising of fiber Bragg grating sensor. PSNR and MSE of fiber Bragg grating sensor after de-noising were shown in table 1. Can be seen from table 1, the outline of the fiber Bragg grating sensor signals after wavelet transform denoising PSNR, said fiber Bragg grating sensor signal quality is better, noise removal more thoroughly, and contrast methods cannot completely eliminate the noise in the fiber Bragg grating sensor signals effectively, the interferences with fiber Bragg grating sensor measurement accuracy, MSE is far less than contrast method at the same time, said contour wavelet transform denoising results more stable, and the method of denoising time is shorter, can satisfy the mass of fiber Bragg grating sensor signal denoising practical application requirements.

Table 1 compares the performance of the classical raster sensing signal de-noising method

\begin{tabular}{ccc}
\hline Denoising method & $P S N R$ & $M S E$ \\
\hline $\begin{array}{c}\text { The wavelet } \\
\text { transform }\end{array}$ & 20.33 & 8.00 \\
$\begin{array}{c}\text { The literature[17] } \\
\text { Contour wave } \\
\text { transform }\end{array}$ & 22.19 & 7.72 \\
& 24.73 & 7.24 \\
\hline
\end{tabular}

\section{Summary}

Serious impact on the quality of the fiber Bragg grating signal noise, its application scope and bring certain actual application value of interference, in order to eliminate the adverse effect, was proposed based on wavelet transform outline of fiber grating sensing signal denoising method, according to outline the multi-scale wavelet transform and directional advantages, the fiber Bragg grating sensor signal decomposition, found in the decomposition of the signal noise data, and through the corresponding filter to remove noise, and through the inverse operation outline of the wavelet transform denoising signal after refactoring, improves the signal-to-noise ratio of the fiber Bragg grating sensor signals, and compared with other denoising method has carried on the experiment. The contrast method can remove some useful signals while removing noise, while the method in this paper may keep the useful signal as much as possible, and at the same time improve the real-time performance of the fiber Bragg grating sensing signal.

\section{Acknowledgement}

Xijing University Scientific Research Foundation Project(XJ140236). 


\section{References}

[1] L Li ,A J Xia : The advantages and applications of fiber Bragg grating sensing technology [J]. Optical communication technology, 2007(7): 62-64.

[2] K.W. Zhang, S.W. Zhang and S. X.Zhao: Application of fiber Bragg grating strain sensor in bridge structure monitoring [J]. Optical instrument, 2014, 36(1):15-19.

[3] A.Q. LI, G.D.ZHOU: Research progress and prospect of optical fiber Bragg sensor testing technology [J]. Journal of southeast university (natural science edition),2009,39(6):1298-1306.

[4] Jeanno F, Joel C, John B: Low energy impact damage monitoring of composites using dynamic strain signals from FBG sensors-part II: damage identification [J]. Composite Structures, 2012,94:593-600.

[5] Z. Q.LIN, H. YANG and H.S. CHEN: Temperature compensation of fiber grating strain sensor [J]. Journal of southeast university (natural science edition), 2007, 37(2): 310-314.

[6] C.X NING, S.S. ZHANG : Experimental study on temperature compensation of fiber grating [J]. Journal of hebei university of science and technology, 2006, 27(2): 155-157.

[7] P.X.ZHENG, Y.L. SONG and D.S. ZHANG: Experimental study on temperature and strain sensing characteristics of fiber Bragg grating [J]. Instrument technology and sensor,2008,22(11):12-15.

[8] X.F. ZHOU,L.LIANG: Experimental study on stability of fiber Bragg grating sensor [J]. Sensor and microsystem, 2007, 26(11): 25-27.

[9] Loutas T H, Kostopoulos V, Ramirez-Jimenez C, et al: Damage evolution in center-holed glass/polyester composites under quasi-static loading using time/frequency analysis of acoustic emission monitored waveforms [J]. Composite Science and Technology, 2006, 66:1366-1375.

[10]L.Q.HOU,X.F.ZHAO;Z.P.LENG and T.SUN :Improved calculation value of temperature compensation of fiber Bragg grating strain sensor [J]. Journal of sensor technology, 2017, 27(1): 70-74.

[11]J.P.LI, X.L. ZENG and J.W. GUAN: Study on refractive index sensing of fiber Bragg grating [J]. Laser and infrared, 2016, 46(6): 737-741.

[12] Hampshire T A, Adeli H.:Monitoring the behavior of steel structures using distributed optical fiber sensors. Journal of Constructional Steel Research, 2000, 53(3): 267-281.

[13]Hill K O, Fujii Y, Johnson D C, et al. Photosensitivity in optical fiber waveguides: Application to reflection filter fabrication[J]. Applied Physics Letters, 2008, 32(10): 647-649.

[14]J. ZHOU, T.G.NING: Study on demodulation of fiber Bragg grating sensing signals [J]. Optical communication technology, 2010,8:8-11.

[15]X.LYU, Z.B.ZHANG and Y. QU: Study on curved long-period photonic crystal fiber grating sensing [J]. Laser technology, 2015, 39(4):571-575.

[16]J. J.CAO, L.L.HU and R. ZHAO: An optical fiber grating sensing signal de-noising method that improves the wavelet threshold function [J]. Journal of sensing technology, 2015, 28(4): 521-525.

[17] Y. P.WANG, L.L.HU and B.WANG: Optical fiber grating sensing signal de-noising based on digital filtering and its FPGA implementation [J]. Journal of xi 'an university of technology, 2015, 31(1): 95-100.

[18]L. LIU, M. YU and R.J. YANG: Wavelet de-noising for optical fiber Raman temperature sensing system [J]. China laser, 2013, 40(6): 1-5. 